\title{
INOVAÇÃO, EMPREENDEDORISMO E NEGÓCIOS TECNOLÓGICOS EM UNIVERSIDADES E INSTITUTOS DE PESQUISA PÚBLICOS -IPPS NO CENÁRIO PÓS-LEI DE INOVAÇÃO, NO BRASIL - UMA BREVE REVISÃO BIBLIOGRÁFICA
}

Innovation, Entrepreneurship and Technological Businesses in Public Universities and Research Institutes in the Scenario Post Innovation Law, in Brazil - A Brief Literature Review

\section{Branca Terra' ${ }^{1}$}

Resumo Objetivos: $O$ presente artigo objetiva desenvolver análises reflexivas sobre as questões relacionadas à inovação, empreendedorismo e negócios tecnológicos no âmbito das universidades e dos institutos de pesquisa públicos - IPPs, nesse cenário brasileiro, pós-lei de inovação. Especificamente, objetiva identificar e analisar características e contextos relacionados ao empreendedorismo e aos negócios tecnológicos públicos, inovadores, bem como a forma de organização de seus planejamentos estratégicos. Materiais e métodos: A metodologia constou de uma breve revisão bibliográfica sobre os temas relacionados à inovação, empreendedorismo e negócios tecnológicos. Resultados: São apresentados conceitos relacionados à inovação, empreendedorismo e negócios tecnológicos, no âmbito da administração pública, passando pelo entendimento de sete temas interligados, escolhidos pela autora: empreendedorismo tecnológico; negócios tecnológicos; universidade empreendedora, hélice tríplice, sistemas regionais de inovação, spin-offs, incubadoras e parques tecnológicos. Conclusão: Uma das contribuições das universidades e institutos de pesquisa públicos para a criação de spin-offs acadêmicos é a adequação da cultura da organização ao planejamento estratégico dos negócios tecnológicos.

Palavras-chave: Empreendedorismo, Inovação, Negócio Tecnológico, Spin-offs.

Abstract Aims: This paper aims to develop critical and reflective analysis on issues related to innovation, entrepreneurship and technological businesses in universities and public research institutes - IPPs, in the Brazilian scenario, post-innovation law. Specifically, it aims to identify and analyze characteristics and contexts related to entrepreneurship and technological businesses public, innovative, and how to organize their strategic planning, and information about the incubators and technology parks as some of the habitats of innovation. Materials and methods: The methodology used is a brief literature review on topics related to innovation, entrepreneurship and technological businesses. Results: The author presents a reflexive analysis on issues related to innovation, entrepreneurship and technological businesses within public administration, through the understanding of seven interconnected themes, chosen by the author: technological entrepreneurship, technological businesses, entrepreneurial university, triple helix, regional innovation systems, spin-offs, incubators and technology parks. Conclusion: One of the contributions of universities and public research institutes for the creation of academic spin-offs is the adequacy of the organization's culture to the strategic planning of technological businesses.

Keywords: Entrepreneurship, Innovation, Technological Business, Spin-offs. 


\section{Introdução e Objetivos}

No Brasil, o atual cenário pós-lei de inovação ${ }^{[1]}$ vem sendo delineado pela implementação de inúmeras ações desenvolvimentistas, focadas na criação de negócios tecnológicos inovadores pelos principais atores da produção de conhecimento (universidades ${ }^{[2]}$, empresas e governos) ${ }^{[3]}$, que atuam nos 8 (oito) mais relevantes contextos de inovação legal, produtivo, científico, tecnológico, financeiro, social, ambiental, logístico.

Estas ações apresentam um amplo espectro, o qual pode ser exemplificado, no contexto legal: pela elaboração de políticas públicas de C,T\&l que incluem, por exemplo, a promulgação das leis estaduais de inovação, bem como, no contexto científico: pelas universidades e institutos de pesquisa (no âmbito das instituições científicas e tecnológicas - ICTs), principalmente as instituições públicas, que vêm se organizando para gerir a transferência de conhecimento para o mercado, por meio da implementação de políticas institucionais.

Pode-se citar, como exemplo: a regulamentação interna desta lei, a mobilização e interação dos atores que compõem sua rede de conhecimento ${ }^{[4]}$, em especial - as estruturas (internas) organizacionais híbridas ${ }^{[5]}$ (in- cubadoras $^{[6]}$, núcleos de inovação tecnológica - NITs ${ }^{[7]}$, grupos de pesquisa "quase firmas"[8], empresas de base tecnológica ${ }^{[9]}$ e spin-offs ${ }^{[10]}$, entre outras) e os professores, alunos, pesquisadores e técnicos que vêm sendo capacitados em atitudes empreendedoras e demais conceitos relacionados à inovação, empreendedorismo e negócios tecnológicos.

O presente artigo objetiva desenvolver análises reflexivas sobre as questões relacionadas à inovação, empreendedorismo e negócios tecnológicos no âmbito das universidades e dos institutos de pesquisa públicos - IPPs, nesse cenário brasileiro, pós-lei de inovação. Especificamente, objetiva identificar e analisar características e contextos relacionados ao empreendedorismo e aos negócios tecnológicos públicos, inovadores, bem como a forma de organização de seus planejamentos estratégicos.

\section{Metodologia}

A metodologia para a elaboração do presente artigo consta de uma breve revisão bibliográfica sobre os temas relacionados à inovação, empreendedorismo e negócios tecnológicos, além de uma análise documental e experiências da autora.

[1] Lei No 10.973, promulgada em 2 de dezembro de 2004 e regulamentada pelo Decreto 5563, de 11 de outubro de 2005, (nota da autora).

[2] As universidades e consequentemente as universidades públicas têm a abrangência de características com os IPPs, (nota da autora).

[3] A interação universidade-empresa-governo é estudada pelo conceito da hélice tríplice, (ETZKOWITZ, 2003).

[4] Redes de conhecimento são espaços onde ocorrem a interação entre atores de diversas áreas, pertencentes ou não a uma mesma organização, (nota da autora).

[5] São as organizações híbridas, que internalizam o relacionamento entre as três esferas (universidade-empresa-governo), estimulando e criando um espaço de interação. (ETZKOWITZ, 2003)

[6] As incubadoras de empresas são infraestrutura para abrigar o empreendedor, (nota da autora).

[7] “A ICT deverá dispor de núcleo de inovação tecnológica, próprio ou em associação com outras ICTs, com a finalidade de gerir sua política de inovação", (nota da autora). [8] Os grupos de pesquisa organizados sob a forma de "quase-firma", onde um professor/pesquisador coordena o trabalho de assistentes e estudantes em treinamento. Ao mesmo tempo em que busca financiamentos para suas pesquisas, publica artigos, elabora relatórios técnicos, entre outros serviços, (ETZKOWITZ, 2003).

[9] Empresas de Base Tecnológica - EBTs são empresas que incorporam, no seu processo produtivo, um elevado grau de conhecimento científico e domínio de técnicas complexas (BAETA, 1997).

[10] É uma: (a) empresa oriunda de laboratório e resultante de pesquisa acadêmica ou industrial; (b) empresa impulsionada por outra já estabelecida no mercado, para atuar na mesma área de negócio, mas com produto ou serviço diferente daquele que a empresa original comercializa, (BOTELHO E ALMEIDA, 2009). 


\section{Uma Breve Revisão Bibliográfica}

A lei de inovação vem sendo considerada no país, como um mecanismo indutor de negócios tecnológico inovadores, principalmente nas universidades e institutos de pesquisa públicos - IPPs, as quais já desempenham um papel de ator econômico e social na atual sociedade do conhecimento ${ }^{[11]}$. Esses atores vêm implementando ações para a construção da cultura empreendedora para alunos, professores, pesquisadores e técnicos dessas instituições públicas como iniciativa estruturante das demandas de desenvolvimento econômico e social atuais do país.

O desenvolvimento de análises reflexivas sobre as questões relacionadas à inovação, empreendedorismo e negócios tecnológicos, no âmbito da administração pública, passa pelo entendimento de sete temas interligados, escolhidos pela autora: 1) empreendedorismo tecnológico; 2) negócios tecnológicos; 3) universidade empreendedora, 4) hélice tríplice, 5) sistemas regionais de inovação, 6) spin-offs, 7) incubadoras e parques tecnológicos, os quais estão a seguir relatados, nessa breve revisão bibliográfica.

\section{Empreendedorismo Tecnológico}

O empreendedorismo é o processo de criação de algo novo e com valor, ao qual é necessária a dedicação de tempo e esforço pessoal, a submissão aos riscos financeiros, psicológicos e sociais, entretanto, o seu êxito resulta em recompensas monetárias e sociais, bem como a satisfação pessoal de independência.

A partir dos estudos de Richard Cantillon (1680-1734) e Jean-Baptiste Say (1767-1832) conforme citado em Baeta e Borges (2006), começou a ser propagada a importância do empreendedorismo, passando pelas considerações de Schumpeter (1883-1950), em sua visão sobre a importância do empreendedorismo na inovação.

Para Shane e Venkataraman (2000), o empreendedorismo está diretamente ligado à identificação e à exploração de oportunidades econômicas: "... envolve o estudo das fontes de oportunidades; os processos de descoberta, exploração, avaliação e exploração de oportunidades; e o conjunto de indivíduos que descobre, como avaliar essas oportunidades e explorá-las...."

Também deve-se ressaltar o conceito de Joseph Schumpeter publicado em seu trabalho de 1934, Economic Reconstruction, que liga o empreendedorismo diretamente a inovação, mas que o empreendedorismo não é limitado, por eles, à inovação.

Nesse contexto das diversas definições e tipos de empreendedorismo, surge a do empreendedorismo tecnológico que segundo Baeta e Borges (2006) tem algumas particularidades em relação ao empreendedorismo tradicional, o que torna tal processo de criação mais difícil.

O empreendedor tecnológico participa da criação de empresas de base tecnológica ${ }^{[12]}$. Baeta e Borges (2006) cita que as empresas de base tecnológica fazem, em geral, parte de uma nova indústria e propõem ao mercado algum tipo de inovação, por isso sofrem com a inexistência no mercado de uma base de conhecimento sólida para o tipo de produto ou ação que a nova empresa está propondo.

Uma série de barreiras estruturais e fatores limitantes constrangem a ação das empresas de base tecnológica no estágio industrial emergente: a ausência de infraestrutura de instalações, de canais adequados de distribuição e suprimento de serviços complementares necessários, a qualidade irregular dos produtos, as dificuldades de obtenção de matérias primas e componentes, a ausência de padronização, escala e

[11] A prestação de serviços é uma atividade da produção que requer o uso cada vez mais intenso de conhecimentos e competências técnicas, (nota da autora).

[12] Empresa de Base Tecnológica - EBT é uma organização que fundamenta sua atividade produtiva na aplicação de conhecimentos científicos e tecnológicos com objetivo de promover a inovação, (nota da autora). 
externalidades de produção, além de um estado de "confusão" (ou às vezes desconfiança) por parte dos clientes e consumidores. Em outra dimensão, há incertezas quanto à imagem e credibilidade das empresas iniciantes junto à comunidade financeira e finalmente há atrasos e transtornos na obtenção de aprovação às regulamentações que pouco a pouco se estabelecem (PORTER, 1986).

A capacitação desses empreendedores passa pelo conhecimento de conceitos teóricos inerentes à gestão e também das ferramentas necessárias a esse fim. Nessas aprendizagens empreendedoras, deve-se fazer com que essas pessoas envolvidas em negócios tecnológicos inovadores, (geralmente pesquisadores, professores e técnicos, os quais em diversas ocasiões são integrantes de grupos de pesquisa), sejam capazes de: 1) entender os conceitos teóricos relativos a negócios tecnológicos; 2) conhecer os contextos onde surgem os negócios tecnológicos; 3) compreender as atitudes empreendedoras, potencializando aspectos cognitivos, emocionais e comportamentais, para uma adquirir uma postura pró-ativa diante na gestão do negócio tecnológico; 4) identificar oportunidades para a concepção negócios tecnológicas; 5) utilizar conceitos fundamentais da administração e práticas estratégicas para uma sólida gestão sustentável da inovação; 6) distinguir práticas de marketing específicas para negócios tecnológicos; 7) planejar, elaborar, acompanhar e avaliar (gestão de negócios tecnológicos) negócios tecnológicos (DORNELAS, 2002); 8) buscar formas de aquisição de recursos para negócios tecnológicos e 9) apropriar e transferir conhecimento e tecnologia para o mercado.

\section{Modelos para o Mercado}

Um negócio tecnológico é identificado a partir de oportunidade surgida durante o desenvolvimento de pesquisa científica e tecnológica, geralmente durante os trabalhos dos laboratórios e grupos de pesquisa, sendo proposto ao mercado por uma EBT, podendo ou não ser uma start up ${ }^{[13]}$, uma spin-off ou uma spin out ${ }^{[14]}$.

Os negócios tecnológicos geralmente são provenientes de inovação. A FINEP, Agência Brasileira de Inovação, ligada ao Ministério da Ciência Tecnologia e Inovação - MCTI ${ }^{[15]}$ adota as definições relacionadas a esse tema determinadas no Manual de Oslo ${ }^{[16]}$.

Hoje, a posição do Brasil no ranque mundial da inovação pode ser observada no Global Innovation Index ${ }^{[17]}$. A edição de 2012 mostra que o Brasil hoje se encontra na 47a posição e incluiu o estudo de 141 (cento e quarenta e uma) economias, que representam $94,9 \%$ da população mundial e 99,4\% do PIB mundial (medido em dólares americanos). O relatório classifica os países com base em suas capacidades de inovação e de resultados usando indicadores chamados de "pilares da inovação": ambiente político, ambiente regulatório, educação, infraestrutura tecnológica e exportações e emprego.

Para que seja realizado um negócio tecnológico é necessário um planejamento. Esse plano de negócios deve estar articulado às estratégias do negócio, basea-

[13] Start up é uma empresa de inovação, cuja base técnica está na pesquisa e desenvolvimento tecnológico, (nota da autora).

[14] Spin out é uma empresa que surge a partir de uma ideia de outra empresa, para explorar novas oportunidades de negócio tecnológico, (nota da autora).

[15] A FINEP é a Financiadora de Estudos e Projetos, ligada ao Ministério da Ciência e Tecnologia - MCT, antigo nome do MCTI.

[16] Lançado pela Organização para Cooperação Econômica e Desenvolvimento, Departamento Estatístico da Comunidade Européia em 1990, a primeira edição denominada "Manual de Oslo - Proposta de Diretrizes para Coleta e Interpretação de Dados sobre Inovação Tecnológica", com o objetivo de orientar e padronizar conceitos, metodologias e construção de estatísticas e indicadores de pesquisa de P\&D de países industrializados. Pode ser encontrado em download.finep.gov.br/ imprensa/manual_de_oslo.pdf, acesso em janeiro de 2013.

[17] É elaborado pelo Instituto Europeu de Administração de Negócios - INSEAD, (sigla em francês) em parceria com a Organização Mundial de Propriedade Intelectual WIPO, (sigla em inglês). O estudo tem como finalidade apontar como os países estão buscando alternativas para resolver principalmente problemas ou desafios globais. Pode ser encontrado em www.globalinnovationindex.org, acesso em janeiro de 2013. 
das em tecnologia e inovação. Hoje o capital de risco, por meio dos capitalistas de risco ${ }^{[18]}$ e as agências de fomento brasileiras (por exemplo: Banco Nacional de Desenvolvimento Econômico e Social - BNDES e a FINEP exigem nos editais os planos de negócios, para a concessão dos financiamentos de projetos de inovação, principalmente os de subvenção econômica, prevista na lei de inovação.

Assim, esse planejamento deve incluir todos os parceiros do projeto e ter na sua estrutura os seguintes elementos constitutivos: sumário executivo; descrição da EBT; descrição do negócio tecnológico; oportunidade do negócio tecnológico; contextos do negócio tecnológico; financiamentos para EBT; planejamento estratégico da EBT; produtos e/ou serviços da EBT; incubadora de empresas onde ficará instalada a EBT; município onde ficará instalada a EBT e os benefícios fiscais (futuro); grupo(s) de pesquisa associado(s) à EBT; plano de P,D\&l da EBT; plano de marketing da EBT; plano Operacional da EBT; plano financeiro da EBT; referências bibliográficas. Em anexo, os empreendedores devem incluir as minutas dos: a) contratos de transferência de tecnologia (patente, licenciamentos e royalties) entre o grupo de pesquisa, o IPP, da incubadora com a EBT, podendo também ser incluídos os órgão financiadores; b) contratos com o (s) órgão (ãos) financiador(es) e capitalistas de risco e o contrato com a incubadora.

\section{Universidade Empreendedora}

O modelo dessas instituições, chamadas de "universidades empreendedoras"[19] está emergindo nos Estados Unidos, América Latina, Europa e Ásia, e vem sendo reconhecida como a universidade do futuro ou universidade do século 21, (CLARK, 1998; SLAUGHTER \& LESLIE, 1999; ETZKOWITZ ET ALL, 2000).

A revisão de literatura sobre os fatores ambientais que afetam a criação e o desenvolvimento das universidades empreendedoras tem início nas considerações de Etzkowitz, (1983) que cita: "universidades são consideradas novas fontes de fundos, tais como patentes, pesquisa sob contratos e parceria com empreendimentos privados".

Para Clark (1998) a universidade empreendedora é vista como característica de um sistema social, e não numa visão de negócios. Significa que a instituição aceitou correr riscos quando iniciou novas práticas. Uma universidade empreendedora busca ser inovadora, persegue uma mudança substantiva no seu caráter organizacional visando alcançar uma nova postura no futuro. Assim, as universidades empreendedoras buscam se tornar stand-up universidades que são atores relevantes por si mesmos. Dentro deste contexto, o empreendedorismo pode é tratado como um processo ao invés de considerado um resultado.

Etzkowitz et all, (2000) voltou a chamar a atenção para o fato de: “... uma universidade empreendedora é um fenômeno global, com uma trajetória de desenvolvimento isomórfico, apesar dos diferentes pontos de atuação e modos de expressão."

No modelo da hélice tríplice a universidade empreendedora é um importante ator do desenvolvimento econômico e social das regiões onde está instalada, (ETZKOWITZ ET ALL, 2000).

\section{Hélice Tríplice}

A hélice tríplice é um modelo conceitual que sugere que há uma nova dinâmica nas relações entre univer-

[18] Capital de risco é uma modalidade de investimento utilizada para apoiar negócios de inovação e funciona pela compra de uma participação acionária, geralmente minoritária, com objetivo de ter as ações valorizadas no futuro, (nota da autora).

[19] Nesse modelo, o termo universidade empreendedora" tem a abrangência para institutos de pesquisa ou "universidades de pesquisa", no contexto americano citado por ETZKOWITZ ET ALL, (2000). 
sidade, indústria e governo e na participação de cada um nas ações de promoção da inovação e da pesquisa científica para gerar novos conhecimentos e tecnologia, (LEYDESDORFF E ETZKOWITZ, 1996). A interação entre universidade, empresa e governo foi ampliada pela introdução de um novo conceito denominado "Triple Helix Spaces"[20]: Conhecimento, Inovação e Consenso, o qual exibe os processos e mecanismos por meio das quais as esferas institucionais interagem e se modificam ao longo do tempo para a consecução das inovações, (ETZKOWITZ E RANGA, 2010).

As incubadoras são exemplos de infraestrutura de interface ideal para a atuação dos atores do modelo de hélice tríplice de relações universidade-empresa - governo, sendo consideradas como organizações híbridas, que internalizam o relacionamento entre as três esferas, estimulando e criando um espaço de interação. A premissa das incubadoras é que a formação de firmas pode ser melhorada ao ser organizada como um processo educacional. No modelo da hélice tríplice as empresas e cooperativas incubadas são vistas como organizações que internalizam o relacionamento entre ciência, empresas e estado, estimulando a criação de um espaço interativo que conecta estas esferas.

Em geral, as incubadoras chamadas de tecnológicas abrigam empresas spin-offs ${ }^{[21]}$ que são EBTs citadas no conceito da hélice tríplice. De acordo com Botellho e Almeida (2009) as empresas spin-off receberam impacto positivo após a provação da lei de inovação pois permite que pesquisadores possam solicitar uma licença das suas atividades acadêmicas para a organização desta modalidade de empresa. Entretanto, a necessidade de regulamentação deste artigo da lei por parte das universidades e a pouca disseminação do conhecimento deste mecanismo jurídico faz com que esta possibilidade seja ainda pouco utilizada.

Na verdade, o que se espera à luz da hélice tríplice é que os grupos de pesquisa de universidade e institutos de pesquisa interajam, por meio da indução da lei de inovação, com as incubadoras e que dessa interação possam surgir EBTs, isto é spín-offs capazes de inserir tecnologia na indústria brasileira e com isso fazer com que o país atinja taxas de crescimento e de índice de desenvolvimento humano ${ }^{[22]}$, além de índices globais de inovação ${ }^{[23]}$, compatíveis com seu PIB ${ }^{[24]}$. Logo, a hélice tríplice recomenda que se observem os grupos de pesquisa chamados de "quase firmas", que funcionam como entidades-empresa dentro das universidades empreendedoras, faltando apenas um lucro direto para torná-los uma empresa.

Resumindo, pode-se considerar os grupos de pesquisa "quase firmas", aqueles que funcionam como organizações lucrativas, faltando apenas um lucro direto para torná-los uma empresa, (ETZKOWITZ, 2003).

\section{Sistemas Regionais de Inovação}

Os sistemas de inovação (segundo Freeman, 1974; Lundvall, 1992; Nelson, 1993; Edquist, 1996) são um conjunto de elementos e estruturas de uma nação, no

[20] Espaços da hélice tríplice, (ETZKOWITZ, 2003).

[21] Spin off é uma empresa criada a partir de uma ideia acadêmica, geralmente em grupos de pesquisa, (nota da autora).

[22] O IDH - Índice de Desenvolvimento Humano oferece um contraponto a outro indicador muito utilizado, o Produto Interno Bruto (PIB) per capita, que considera apenas a dimensão econômica do desenvolvimento. Criado por Mahbub ul Haq com a colaboração do economista indiano Amartya Sen, ganhador do Prêmio Nobel de Economia de 1998, o IDH pretende ser uma medida geral, sintética, do desenvolvimento humano", encontrado no Programa das Nações Unidas para o Desenvolvimento - PNUD, no Relatório do Desenvolvimento Humano - Crescimento Econômico e Desenvolvimento Humano, 1996 em www.pnud.org.br, acesso em janeiro de 2013.

[23] The Business School for the World - INSEAD e a Organização Mundial da Propriedade Intelectual - WIPO, agência das Nações Unidas co-publicam o Índice Global de Inovação (GII) desde 2012, encontrado em www.insead.edu, acesso em janeiro de 2013.

[24] O PIB - produto interno bruto representa a soma (em valores monetários) de todos os bens e serviços finais produzidos numa determinada região (quer sejam países, estados ou cidades), durante um período determinado (mês, trimestre, ano, etc). O PIB é um dos indicadores mais utilizados na macroeconomia com o objetivo de mensurar a atividade econômica de uma região, (nota da autora). 
caso de sistemas nacionais, que assumem funções específicas no processo de produção, transmissão e armazenamento de conhecimentos para fins de inovação. Tais sistemas são caracterizados por sua capacidade de interação, mediante a articulação desses elementos, para a produção de conhecimento, sua difusão e utilização, de forma competitiva e lucrativa.

Sistema nacional de inovação, segundo relato de Albuquerque, (2004) é um conceito síntese da elaboração evolucionista neo-shumpeteriana: ele expressa o complexo arranjo institucional que, impulsionando o progresso tecnológico, determina a riqueza das nações. Denomina-se sistema regional ou local de inovação quando se realiza a análise e contextualização de uma região de um sistema nacional de inovação. Entretanto, não se pode desconsiderar a influência do sistema nacional de inovação sobre o sistema regional. O sistema nacional de inovação é um conceito importante para compreender o papel das universidades e dos IPP no avanço tecnológico dos países.

\section{Spin-off}

Diversos autores definem 'spin-off' como uma empresa com produtos ou serviços desenvolvida a partir de uma idéia tecnológica ou de conhecimento técnico/científico originado por um membro da universidade ou uma empresa criada para explorar a propriedade intelectual IP derivada de uma atividade de pesquisa desenvolvido em uma instituição acadêmica.

Segundo Botelho e Almeida (2009), spin-off as empresas receberam no Brasil, um impulso com a introdução da Lei de Inovação, uma vez que permite que os pesquisadores tiram licença de suas atividades acadêmicas, a fim de criar um negócio desses. No entanto, o fato de que as universidades ainda precisam resolver seus regulamentos no que diz respeito à lei e que o conhecimento deste mecanismo legal recebeu difusão limitada significa que ela ainda é uma opção pouco usada.

\section{Incubadoras e Parques Tecnológicos - PqTs}

As incubadoras e parques tecnológicos estão incluídos nos chamadas de habitats de inovação. Um habitat de inovação é o "locus" promotor de inovação ou ainda, são ambientes propícios ao desenvolvimento contínuo de inovações tecnológicas. Funcionam como espaços de aprendizagem coletiva, de intercâmbio de conhecimentos e práticas produtivas, de interação entre os diversos agentes de inovação. Outras estruturas também são consideradas habitats de inovação: tecnópole ou tecnópolis ou polos ${ }^{[25]}$; arranjos produtivos locais - APLs $s^{[26]}$; clusters $^{[27]}$; hotéis de ideias e projetos, hotéis tecnológicos, aceleradoras de empresas ${ }^{[28]}$; consórcios de empresas inovadoras $^{[29]}$; redes de inovação ou de desenvolvimento ${ }^{[30]} \mathrm{e}$ rotas tecnológicas ${ }^{[31]}$.

[25] Regiões de cidades onde é evidente a articulação dos atores locais com vistas ao desenvolvimento tecnológico regional, baseado numa estratégia de desenvolvimento inovadora, (nota da autora).

[26] São clusters de empresas localizadas em uma mesma região, que atuam na mesma cadeia produtiva o correlatas e mantêm interação, (nota da autora).

[27] São polos produtivos reconhecidos pal interação entre empresas de determinado setor econômico, (nota da autora).

[28] São locais com programas de pré-incubação, isto é, de incentivo para o surgimento de EBTs (nota da autora).

[29] São associações de pequenas empresas organizadas de forma contratual, que se unem para viabilizar soluções econômicas, sociais e investimentos planejados, na maioria das vezes na fase pré-competitiva dos projetos.

[30] A rede de inovação ou de desenvolvimento é uma rede especifica que promove cultura inovadora entre os atores regionais e aumenta o capital social local, para que ocorra o desenvolvimento econômico e social, por meio da inovação.

[31] Regiões cercadas por infraestrutura logística que incentiva a instalação de EBTs. Geralmente são localizadas próximas a universidades grandes empreendedoras tal como a Route 128 ao redor do Massachusetts Institute of Technology - MIT, em Boston, nos Estados Unidos. 
Conforme citado em Dornelas (2002) o movimento de incubadoras de empresas sempre esteve relacionado ao movimento de empreendedorismo em todos os países onde a criação de empresas e o suporte aos empreendedores, têm sido enfatizados. No Brasil, a primeira incubadora de empresas surgiu muito tempo depois, em 1985, na cidade de São Carlos em São Paulo.

$\mathrm{O} \mathrm{MCT}^{[32]}$ lançou em 2000 um manual para as incubadoras brasileiras sendo que elas atuam em Programas de Pré-incubação, Incubação e Pós-incubação de EBTs. $\mathrm{Na}$ fase de pré-incubação as incubadoras apoiam ideias inovadoras, sem a necessidade de formalização da EBT e tem como objetivos: despertar o espírito empreendedor na comunidade; promover ideias empreendedoras que surjam entre os alunos, professores e técnicos, ajudando-os a amadurecer seus projetos; aumentar a empregabilidade mostrando aos empreendedores outras possibilidades profissionais e proporcionando um diferencial competitivo em suas carreiras; promover as atividades empreendedoras (Feira Tecnológica, Concurso de Plano de Negócio, Seminário de Iniciação Científica, entre outros); aumentar e qualificar a demanda de projetos inovadores para as incubadoras regionais; difundir a cultura empresarial e modernos instrumentos de gestão; contribuir para o desenvolvimento social e tecnológico do país através da criação de novas e inovadoras empresas de base tecnológica, entre outros.

Na fase de incubação as incubadoras apoiam as EBTs nascentes auxiliando no desenvolvimento do negócio tecnológico, tendo objetivos, para os empreendedores: o aumento da: capacitação técnica e administrativa; ao auxílio à elaboração dos documentos de formação e à legalização da EBT; o acompanhamento sistemático ou assessoria para inserção e manutenção da EBT no mercado e conquista da autonomia; a avaliação do grau de autonomia dos empreendedores, entre outros.
E finalmente, na fase de pós-incubação, as incubadoras auxiliam na manutenção do vínculo da EBT graduada com a organização de origem, mesmo ela já estando localizada em um parque científico ou tecnológico.

No manual lançado em 2000 pelo MCT, (hoje MCTI), anteriormente citado, incubadoras podem ser de três tipos diferentes: 1) incubadora de EBT; 2) incubadora de Empresas dos Setores Tradicionais; 3) incubadora de Empresas Mista.

De acordo com Almeida (2004) a incubadora tecnológica é o resultado da bi-evolução da universidade, bem como consequência da ampliação de sua missão e de seu foco para o desenvolvimento econômico. As incubadoras tecnológicas aumentam a taxa de inovação industrial, através da aproximação que constroem entre empresas inovadoras e o mercado, assim como entre as empresas inovadoras e a pesquisa básica e aplicada. As incubadoras situadas nas universidades facilitam a interação das empresas incubadas com a pesquisa, devido à proximidade com os pesquisadores, os laboratórios e as bibliotecas.

Já o movimento dos parques tecnológicos - PqTs, no mundo tem sua gênese com a experiência do Stanford Industrial Park, conhecido como 'Vale do Silício'. Em 1951, nasceu o Stanford Industrial Park, criado a partir do crescimento do número de empresas nascidas da Universidade de Stanford, por incentivo do diretor do centro de Engenharia, Frederick Terman. O parque nasceu a partir do conjunto de condicionantes favoráveis ao desenvolvimento da relação entre as zonas industriais (iniciativa privada), universidades (Universidade de Stanford) e instituições de pesquisa (ETZKOWITZ ET RANGA 2010).

De acordo com De Sá, (2011), entre as décadas de 1960 e 1970, ainda como ações deliberadas e com o intuito de se promover o desenvolvimento econômico local/regional, novos PqTs foram criados, espelhando-se na bem-sucedida experiência do Stanford Industrial Park:

[32] Manual para a Implantação de Incubadoras de Empresas. Coordenação de Sistemas Locais de Inovação. Novembro de 2000. Disponível em http://mct.gov.br/setec/ setec/html, acesso em janeiro de 2013 
Research Triangle Park (Carolina do Norte - EUA), Route 128 (Boston - EUA), Heriott-Watt Park (Edimburgo - Reino Unido), Cambridge Science Park (Cambridge - Reino Unido), Sophia-Antipolis (Nice - França), Grenoble-Meylan (Meylan - França); Daedeok Park (Daedeok - Coréia do Sul).

No Brasil, é tardio o início do movimento de PqTs que se deu em 1984, com a criação de um programa do Conselho Nacional de Desenvolvimento Científico e Tecnológico - CNPq para apoio aos PqTs. O intuito do programa era a criação de EBTs por meio da transferência de conhecimento das pesquisas universitárias. No entanto, em decorrência da falta de descontinuidade de ações, da incipiente capacidade científico-tecnológica, da ausência de políticas específicas para apoio à iniciativa, da carência de uma cultura baseada em inovação e da resistência de parte dos ambientes acadêmico- universitários, a maioria das experiências pioneiras acabou atuando apenas como estruturas de incubação, isto é, as incubadoras de empresas, (DE SÁ, 2011).

Em 1987 foi criada a Associação Nacional de Entidades Promotoras de Empreendimentos Inovadores ANPROTEC, mas somente a partir de meados da década de 1990 e início dos anos 2000, os PqTs inseridos na agenda das políticas brasileiras de ciência, tecnologia e inovação (C,T\&l).

Várias são as terminologias utilizadas, segundo cita De Sá, (2011): (i) Parque Tecnológico, (ii) Parque Científico, (iii) Parque de Inovação, (iv) Parque de Pesquisa, (v) Parque Científico e Tecnológico, (vi) Parque Empresarial, (vii) Parque de Negócios, (viii) Tecnópole, entre outros.

Na prática, os termos Science Park (Parque Científico) e Technopole (Tecnópole) são mais comumente usados na Europa; o termo Technology Park (Parque Tecnológico) prevalece na Ásia, enquanto que a expressão Research Park (Parque de Pesquisa) é comumente utilizada nos EUA e Canadá. Assim como a terminologia, há diferentes propostas para a conceituação de PqTs, com definições sugeridas por pesquisadores no tema e, também, por entidades que possuem programas, estudos ou financiamentos dedicados a PqTs.

\section{Conclusões}

A cultura das universidades e IPPs, isto é: as crenças dos professores, pesquisadores, alunos, técnicos, líderes dos grupos de pesquisa, gestores e suas percepções a respeito do envolvimento da universidade e IPPs com as empresas e governos, em projetos de transferência de conhecimento, contemplados pela lei de inovação deve ser adequada à negócios tecnológicos e utilizada como diretriz para as estratégias das políticas institucionais e para as políticas públicas indutoras de negócios tecnológicos ou spin-offs acadêmicos.

Há necessidade de se obter, a partir dos dados coletados sobre as diferentes culturas organizacionais, informações não apenas sobre os atores da rede de conhecimento dessas organizações, mas também sobre as relações entre esses atores e os atores dos diferentes contextos que interagem com as universidades e IPPs.

Essas informações poderão aumentar o conhecimento institucional sobre os benefícios gerados para a sociedade a partir dos resultados da interação da rede de conhecimento institucional com as incubadoras, parques tecnológicos, empresas e outros parceiros; as formas de parcerias dos atores que produzem conhecimento com empresas; a vocação dos grupos de pesquisa das universidades e IPPs para a criação de tangíveis e intangíveis (marcas patentes, cultivares, softwares, mapas, entre outros) em função das áreas de conhecimento a que pertencem e dos setores produtivos onde atuam; a forma de distribuição dos resultados da pesquisa entre os atores participantes dos negócios tecnológicos e as estratégias de comercialização do conhecimento estabelecidas pelos atores envolvidos nos negócios tecnológicos. 
Como conclusão, pode-se entender que a inovação pode gerar negócios tecnológicos inovadoras desde que sejam implementadas ações empreendedoras relacionadas: à educação para empreender; à conscientização da sociedade sobre a importância da geração e apropriação de ideias; ao crescimento da base brasileira de pesquisadores, ao reconhecimento internacional do país, por sua vocação em algumas áreas do conhecimento; ao crescimento dos investimentos em C,T\&l; à implementação do marco regulatório relacionado à inovação (incluindo incentivos fiscais; disseminação da cultura da inovação; desenvolvimento da indústria de capital de risco; aumento do investimento em inovação por parte das empresas; diminuição da burocracia tempo de registro de empresas, custos de abertura de empresas, entre outros fatores; ao aumento da interação universidade-empresa-governo; ao desenvolvimento de competências para a inovação e principalmente, ao fortalecimento da ética nas relações de ensino, pesquisa e extensão.

\section{Referências}

1. Etzkowitz, H. Research groups as 'quasi-firms': the invention of the entrepreneurial university. Research Policy 32; 109-121; 2003

2. Baeta, A. M. C. As Incubadoras de Empresas de Base Tecnológica: Uma Nova Pratica Organizacional para a Inovação. Tese de Doutorado. COPPE/UFRJ, Programa de Engenharia de Produção, 1997

3. Botelho, A. J. J. ; Almeida, M.. Overcoming Institutional shortcomings for academic spin-offs policies in Brazil. In: VII Triple Helix Conference, 2009, Glasgow. Annals Triple Helix International Conference, 2009.

4. Shane. S. e Venkataraman, S. The Promise of Entrepreneurship as a Field of Research Academy of Management Review, vol. 25, n 1, p 217-228, 2000.
5. Baeta, A. M. C.; Borges, C. V.; Tremblay, D. Empreendedorismo nas incubadoras: Reflexões sobre tendências atuais. Revista Comportamento Organizacional e Gestão, Vol. 12, N.o 1, 7-18, 2006

6. Porter, M. Competition in Global Industries, Harvard Business School Press, Boston, 1986 7. Dornelas, José Carlos Assis. Planejando incubadoras de empresas: como desenvolver um plano de negócios para incubadoras. Rio de Janeiro: Editora Campus, 2002.

8. Clark, B. R. Creating Entrepreneurial Universities: Organization Pathways of Transformation. In: Introduction. Kidlington, Oxford OX5 IGB, UK, p.xiii-xvi, 1998.

9. Slaughter, S.; Leslie, L. L. Academic Capitalism: Politics, Polices and the Entrepreneurial University. In: Chapter One. The Johns Hopkins University Press, p.1-6, 1999.

10. Etzkowitz, H., Webster, A., Gebhardt, C., and Terra, B. R.. The future of the University and the University of the future: evolution of ivory tower into entrepreneurial university. Research Policy, 29: 313-30, 2000

11. Etzkowitz, H.. Entrepreneurial Scientists and Entrepreneurial Universities in American Academic Science. Minerva, 21(2-3): 198-233, 1983.

12. Leydesdorff, L. \& Etzkowitz, H. The Triple Helix-University, Industry, Government Relations: A Laboratory for Knowledge Based Economic Development. publicado nos Anais da "The Triple Helix of University-Industry-Government Relations: The Future Location of Research Conference", Amsterdam, 1996.

13. Etzkowitz, H.; Ranga M. 2010. A Triple Helix System for Knowledge-based Regional Development: From "Spheres" to "Spaces". Theme paper for Triple Helix 8 International Conference, Madrid, October 2010, http://www.triplehelix8.org/downloads/ThemePaper.pdf. Accessed on September, 25, 2010 .

14. Freeman, C. The Economics and Industrial Innovation. Harmondsworth: Penguin, 1974. 15. Lundvall, B. A. National Systems of Innovation - Toward a Theory of Innovation and Interactive Learning. A Cassel Imprint. London. England, 1992.

16. Nelson, R. R. National Innovation Systems - A Comparative Analysis. Oxford University Press. New York, USA, 1993.

17. Edquist, C. Systems of Innovation - Technologies, Institutions and Organizations. A Cassel Imprint. London, England; 1997

18. Albuquerque, E. M. Idéias Fundadoras. Revista Brasileira de Inovação, Volume 3 , Número 1, janeiro/junho, ISSN 1677-2504. Publicada pela Financiadora de Estudos e Projetos - FINEP (www.finep.gov.br), 2004

19. Almeida, Mariza Costa. A Evolução do Movimento de Incubadoras no Brasil. 2004 174 p. Tese de Doutorado cm Engenharia de Produção. COPPE/ UFRJ, Universidade Federal do Rio de Janeiro, Rio de Janeiro, Brasil, 2004.

20. De Sá, Mohana Faria. Avaliação de Práticas de Gestão do Conhecimento de Parques Tecnológicos: Uma Proposta para Apoio à Gestão Pública. 2011, 155f. Tese de Doutorado em Engenharia e Gestão do Conhecimento. Programa de Pós-Graduação em Engenharia e Gestão do Conhecimento da Universidade Federal de Santa Catarina, Florianópolis, Santa Catarina, Brasil, 2011 